\title{
Magnetic interaction at the interface of epitaxial manganite film and $\left(\mathrm{TbCo}_{2} / \mathrm{FeCo}\right)_{n}$ superlattice
}

\author{
Alexander S. Grishin ${ }^{1,2 *}$, Gennady A. Ovsyannikov ${ }^{1}$, Alexey A. Klimov ${ }^{1,2}$, Viktor V. Demidov ${ }^{1}$, Karen Y. \\ Constantinian $^{1}$, Igor V. Borisenko ${ }^{1}$, Vladimir L. Preobrazhensky ${ }^{3,4}$, Nicolas Tiercelin ${ }^{4}$, Phillipe Pernod ${ }^{4}$ \\ ${ }^{1}$ Kotel'nikov Institute of Radio Engineering and Electronics Russian Academy of Sciences, 11 Mokhovaya str., 125009 Moscow, \\ Russia \\ ${ }^{2}$ Moscow Technological University MIREA, 86 Vernadsky av., 119571 Moscow, Russia \\ ${ }^{3}$ The Wave Research Center, Prokhorov General Physics Institute Russian Academy of Sciences, 38 Vavilova str., 119991 Moscow, \\ Russia \\ ${ }^{4}$ Univ. Lille, CNRS, Centrale Lille, ISEN, Univ. Valenciennes, UMR 8520 - IEMN, F-59000 Lille, France
}

\begin{abstract}
Hybrid magnetic heterostructures made of epitaxial manganite $\mathrm{La}_{0.7} \mathrm{Sr}_{0.3} \mathrm{MnO}_{3}$ thin film and intermetallic superlattice $\left(\mathrm{TeCO}_{2} / \mathrm{FeCo}\right)_{n}$ were prepared on orthorhombic $\mathrm{NdGaO}_{3}$ substrates and characterized by means of magneto-optical Kerr effect and magnetoresistance. Experimental data show that magnetic interaction between $\mathrm{La}_{0.7} \mathrm{Sr}_{0.3} \mathrm{MnO}_{3}$ and $\left(\mathrm{TeCo}_{2} / \mathrm{FeCo}\right)_{\mathrm{n}}$ is of the antiferromagnetic type. The features observed in magnetotransport characteristics are caused by the magnetization reversal at the interface between manganite thin film and intermetallic superlattice.
\end{abstract}

\section{Introduction}

The development of spintronic devices based on nanoscale interfaces of magnetic materials is a challenging problem. Stacks of complex materials (manganites, multiferroics, rare earth compounds and others) for non-volatile memory devices were reviewed in [1]. Manipulation of phase transition in manganite $\mathrm{La}_{0.7} \mathrm{Sr}_{0.3} \mathrm{MnO}_{3}$ (LSMO) thin film was achieved by applying positive/negative voltage over an additional gate electrode. Large tunneling resistance effect, accompanied with a moderately magnetoresistance was reported for $\mathrm{LSMO} / \mathrm{BaTiO}_{3} / \mathrm{Co}$ junctions [2].

Recently a promising solution was proposed for a memory cells providing reliable spin manipulation and switching of magnetization between two stable positions in intermetallic structures [3]. The solution is based on a superlattice of exchange-coupled rare-earth compound $\mathrm{TbCO}_{2}$ and $3 \mathrm{~d}$ transition metal FeCo nanolayers. The structure is characterized by a giant magnetostriction and demonstrates spin reorientation transitions by means of an external magnetic field, and/or elastic strain [4,5]. In transition metal oxides $\mathrm{R}_{1-x} \mathrm{~A}_{x} \mathrm{MnO}_{3}$ (manganites) where $\mathrm{R}$ is one of the rare earth elements La or Pr and $\mathrm{A}$ is one of the alkaline earth metals $\mathrm{Sr}$ or $\mathrm{Ca}$, new electronic states and magnetic phases can arise under electric fields and strains in thin film or at the interfaces with dielectrics or other oxides.

However, the magnetic interaction and electronic and spin states at the interface between manganites and intermetallic structures were not studied yet. In this paper, we fabricated manganite/intermetallic heterostructures in order to determine magnetization behavior at interface and to reveal the influence of spin-polarized current on magnetotransport characteristics.

\section{Sample fabrication and experimental}

The heterostructures under consideration consist of two layers. The top layer is the rare earth intermetallic superlattice, consisting of an exchange-coupled multilayered $\left(\mathrm{TbCo}_{2}(4 \mathrm{~nm}) / \mathrm{FeCo}(4 \mathrm{~nm})\right) \mathrm{n}(\mathrm{TCFC})$ stack with $n=25$. The bottom layer is the lanthanum - strontium manganite $\mathrm{La}_{0.7} \mathrm{Sr}_{0.3} \mathrm{MnO}_{3}$ (LSMO) $30 \mathrm{~nm}$ thick film which was epitaxially deposited on an orthorhombic $\mathrm{NdGaO}_{3}(\mathrm{NGO})$ substrate.

The LSMO films were deposited onto $5 \times 5 \times 0.5 \mathrm{~mm}^{3}$ (110) NGO substrates by magnetron sputtering at 700$750^{\circ} \mathrm{C}$ temperature and partial oxygen pressure between 0.1 and $0.3 \mathrm{mbar}$. The desired crystal structure of the LSMO film was ensured by the NGO substrate that promoted the dominance of uniaxial in-plane magnetic anisotropy [3]. The intermetallic superlattice was obtained by the sequentially sputtering of $\mathrm{TbCo}_{2}$ and FeCo nano-layers under applied magnetic field directed in the plane of the substrate, which defined the direction of the uniaxial easy axis magnetic anisotropy. The strength of the magnetic anisotropy in the TCFC superlattice is controlled by the ratio of $\mathrm{Tb}, \mathrm{Co}, \mathrm{Fe}$ elements in the layers as well as by the thickness and number $n$ of $\mathrm{TbCo}_{2}$ and FeCo layers. The heterostructures were fabricated with directions of easy axis magnetization of the manganite and intermetallic superlattice being in parallel. The LSMO film covers the whole $5 \times 5 \mathrm{~mm}^{2}$ area of substrate, whereas the TCFC is a $3 \times 3 \mathrm{~mm}^{2}$ square at the center of the sample (Fig. 1).

Magnetic parameters, the coercive force $\left(H_{c}\right)$ and saturation field $\left(H_{s}\right)$ of heterostructures were studied using magneto-optical Kerr effect (MOKE). The magnetooptical setup involved a semiconductor laser operating at $\lambda=0.63 \mu \mathrm{m}$ wavelength and power $P=5 \mu \mathrm{W}$, a beamsplitter glass plate for forming the reference and signal 
beams, a $\lambda / 2$ thick phase-shifter plate for selecting the $s$ or $p$-type polarization of the incident beam, and a polarizer/analyzer for selecting the signal caused by the meridional Kerr effect. The laser spot diameter at the measuring point is about $1 \mathrm{~mm}$. The investigated sample was placed on a rotary table in the gap of electromagnet. The angle $(\delta)$ of polarization plane rotation of the beam reflected from the surface of the magnetized sample was determined by the compensation method using the polarizer-analyzer. The dependence $\delta(\mathrm{H}) / \delta_{\mathrm{s}} \propto \mathrm{M}(\mathrm{H}) / \mathrm{MS}$ was measured, where $\delta_{\mathrm{s}}$ is the magneto-optical Kerr effect (MOKE) signal value at $\mathrm{M}=\mathrm{MS}\left(M_{S}\right.$ is the saturation magnetization of the sample). A detailed description of the experimental setup is given in [3]. For heterostructures characterization, we also used the vibrating sample magnetometer (VSM). Magnetoresistance $R(H)$ dependencies were obtained by measuring the differential resistance $R=d V / d I$ as a function of the applied magnetic field $H$. Most of measurements were carried out at room temperature $T=300 \mathrm{~K}$.

The Curie temperatures $\left(T_{C}\right)$ for both TCFC and LSMO films were well above $300 \mathrm{~K}$. Nevertheless, some of the resistive characteristics for higher accuracy were measured at $77 \mathrm{~K}$ by a 4-point method.

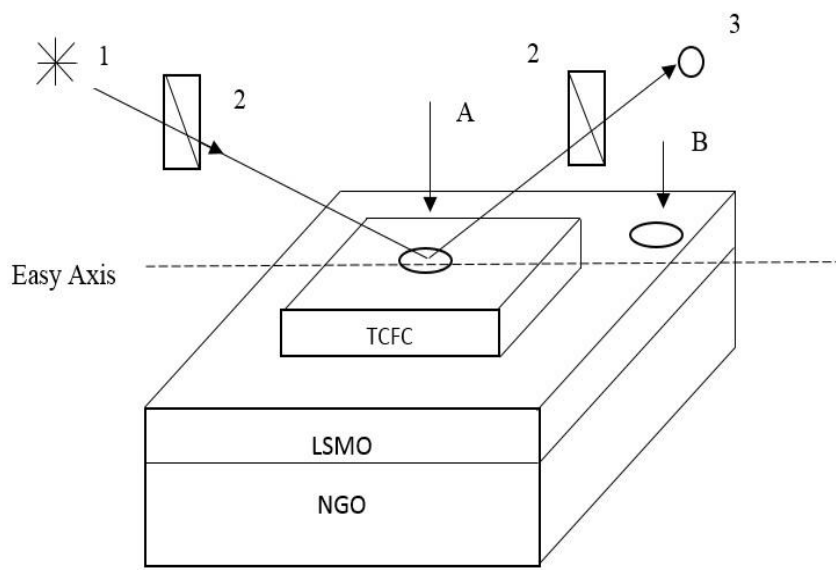

Fig. 1. Schematic view of the investigated heterostructures and Kerr-effect geometry: He-Ne laser (1), polarizers (2), detector (3). "A" and "B" are respectively the spot positions where TCFC/LSMO structure and LSMO film only were probing.

\section{Results and discussion}

\subsection{Kerr effect measurements}

Figure 2 shows the magnetic field dependence of magnetization that was obtained by MOKE measurements when an external magnetic field was directed along the hard axis of magnetization. The magnetization behavior for the TCFC/LSMO heterostructure (the dashed line in Fig. 2b) was obtained when the probing laser at the central part of heterostructure (spot A in Fig.1) whereas the LSMO film was probed when beam was focused at spot B (the solid line in Fig.2b).

It is seen that the saturation field for TCFC/LSMO, $H_{S}$ $\approx 1000$ Oe was much higher than for the LSMO film, $H_{S}$

$\approx 20 \mathrm{Oe}$, and that the saturation magnetization of
TCFC/LSMO heterostructure is also much higher than the one of LSMO film. Note, the influence of the underlying LSMO film cannot be observed by MOKE at the spot A, since the laser beam is screened by the TCFC film. When probing the LSMO film only (spot B), a change of magnetization sign was observed that points to an antiferromagnetic magnetic ordering at the TCFC/LSMO interface of the heterostructure.
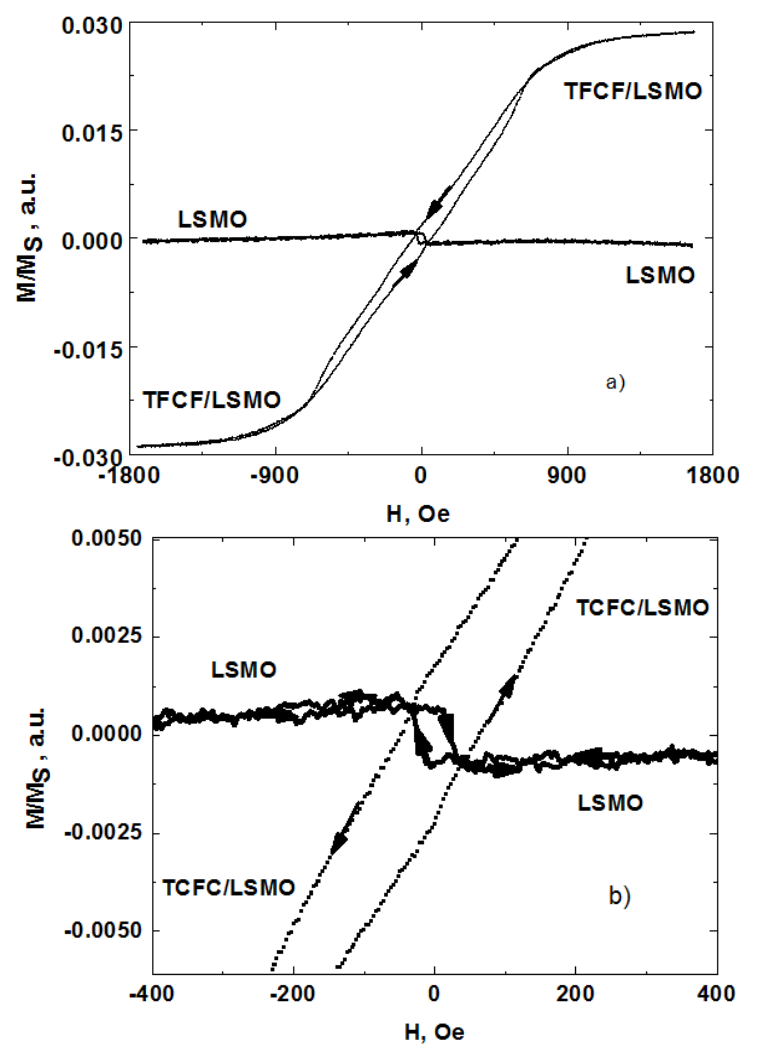

Fig. 2. Magnetization curves of the TCFC/LSMO heterostructure. a) Full-scale plot of magnetization curve for a $\pm 2 \mathrm{kOe}$ sweeping range. Spot position (A) TCFC/LSMO and (B) LSMO film only (see Fig.1). The external magnetic field is directed along the hard magnetization axis (ha) as shown on inset; b) Magnified central part of the magnetization curves for both TCFC and LSMO film with $\mathrm{a} \pm 400$ Oe sweeping range. The arrows indicate the magnetic field evolution.

Figure 3 shows hysteresis loops measured at $H$-field directed along the easy axis. Figure 3 a shows the hysteresis loop for LSMO film and LSMO/TCFC heterostructure when remagnetization range was larger than $2 \mathrm{kOe}$. The widths of the hysteresis loop for both the TCFC/LSMO heterostructure and the LSMO film approximately correspond to a coercive force of about 200 Oe. From Fig. $3 b$ it is seen in a shorter remagnetization range, less than $400 \mathrm{Oe}$, the hysteresis loop of the TCFC/LSMO heterostructure exhibits a $M(H)$ dependence with an inverted sign as in the case when magnetic field was directed along the hard axis. The hysteresis loop width is approximately equal to the coercitivity of the LSMO film obtained before TCFC deposition on top. When $H$-field sweeping amplitude was larger than 400 Oe, the magnetization curve for LSMO film repeats the shape of the hysteresis loop of the TCFC 
superlattice. Increasing the magnetic field sweeping range over 400 Oe leads to a magnetization curve of the LSMO film that coincides with the one shown in Figure 3a.

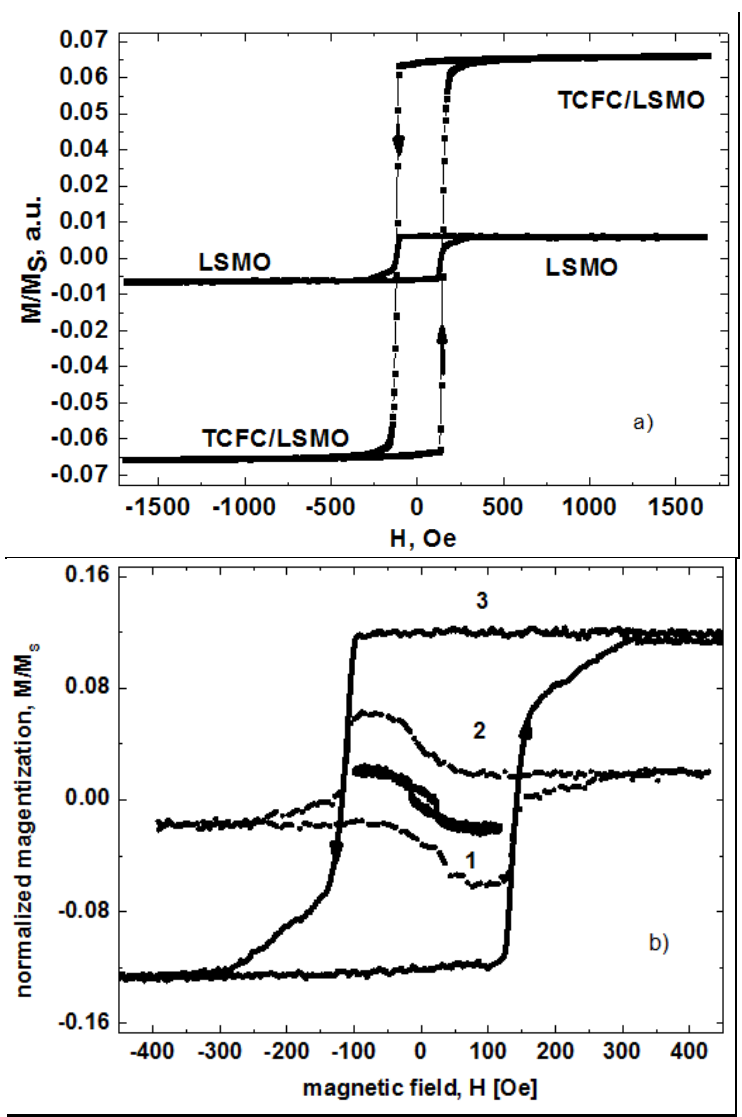

Fig. 3. Magnetization versus magnetic field for the TCFC/LSMO heterostructure. Magnetization normalized for TCFC/LSMO part of the heterostructure. a) Full-scale magnetization curve for $\pm 2 \mathrm{kOe}$ sweep ranges. The external magnetic field is directed along the easy magnetization axis (ea). b) Magnified central part of the magnetization curve for the LSMO film for various sweep ranges:(1) $\pm 100 \mathrm{Oe},(2) \pm 400 \mathrm{Oe}$ and (3) $\pm 2 \mathrm{kOe}$. The arrows indicate the direction of the magnetic field evolution.

\subsection{Magnetization}

Fig. 4a displays VSM-measured the hysteresis loops of single LSMO film deposited on NGO. Hysteresis loops are given for two orthogonal orientations of magnetic field. The saturation magnetic field and magnetization are equal to $H_{S}=20 \mathrm{Oe}, M_{0}=198 \mathrm{emu} / \mathrm{cm}^{3}$, correspondingly. The coercive force and saturation magnetic field are close to each other.

The VSM-measured hysteresis loops of TCFC/LSMO heterostructure for two orthogonal orientations of magnetic field are shown in Fig. 4b. Along the easy axis, the hysteresis curve exhibits two steps corresponding to the reversal of magnetization in the soft (LSMO) and the hard (TCFC) magnetic layers. A similar behavior of magnetization was obtained in the [6] where computer simulations were performed for trilayer magnetic structures taking into account the coupling between layers. In our case, the third layer providing the interaction of two ferromagnetics is the interface of LSMO/TCFC. The thickness of the active layers can be estimated using the approach in the work [7]. As it follows from the magnetization curves, (Fig. 4b) a stable antiferromagnetic (AFM) configuration takes place at the certain range of the external magnetic field. Taking into account the magnetization distributions discussed in [6], we can conclude that the reversal process proceeds more uniformly in the hard magnetic layer than in the soft one. In the former case, the magnetization undergoes a discontinuous change, with its distribution being relatively uniform before the switching. In contrast, the soft magnetic layer is switched very non-uniformly. The non-uniformity expands gradually, moving from the edges of the layer towards its center.
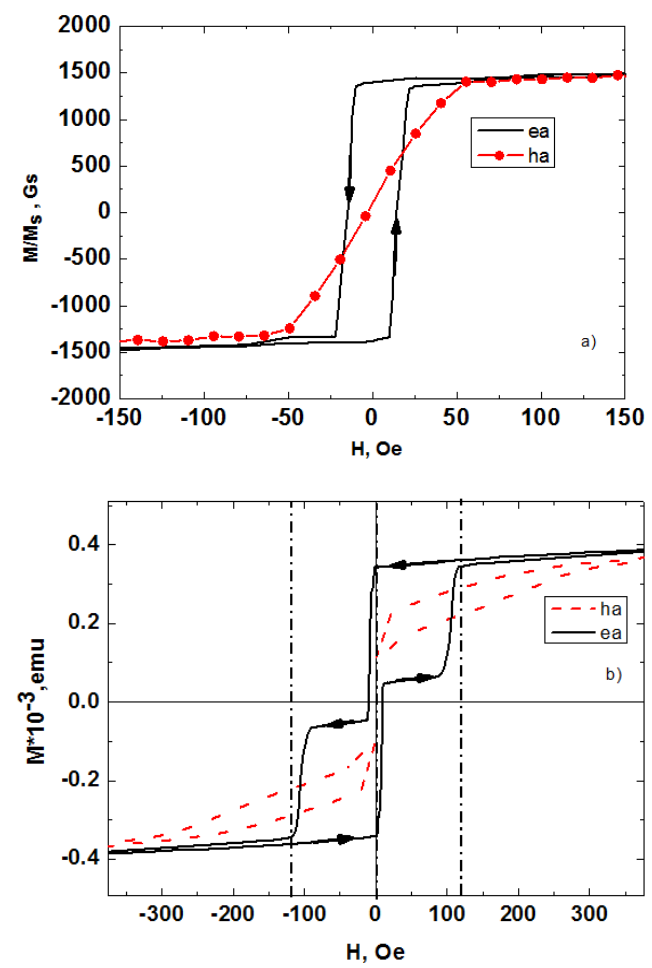

Fig. 4.Hysteresis loops for two structures: a) LSMO film, b) TCFC /LSMO heterostructure. The external magnetic field is directed along the hard axis (dashed lines ha) and along the easy axis (solid lines ea).

\subsection{Magnetoresistance}

The magnetoresistance of the TCFC/ LSMO heterostructure was measured at $T=77 \mathrm{~K}$ for two directions of the applied magnetic field. The variation of magnetoresistance was about $0.2 \%$ at $T=77 \mathrm{~K}$ but it was smaller at $T=300 \mathrm{~K}$. The resistance was measured over the diagonal direction of the substrate, which corresponds to the magnetic hard axis. The magnetic field was applied either along hard magnetization axis (Fig.5a) or along easy magnetization axis (Fig.5 b) of TCFC layer.

At low field there is a shift of the maximum of $R(H)$ which indicates the presence of ferromagnetism. The distance between the peaks of $R(H)$ allows to estimate the 
coercive force of the heterostructure. The slope of the $R(H)$ curves indicates the presence of colossal magnetoresistance in the manganite film. The additional features of magnetization are observed in the magnetic field ranged 250- 500 Oe applied along hard axis. They are probably caused by the switch of magnetic moment in the TCFC superlattice. When the magnetic field is applied along the easy axis $R(H)$ maximum is also shifted about 80 Oe from $H=0$. But there are no features in the range from 250 to 500 Oe of external magnetic field.
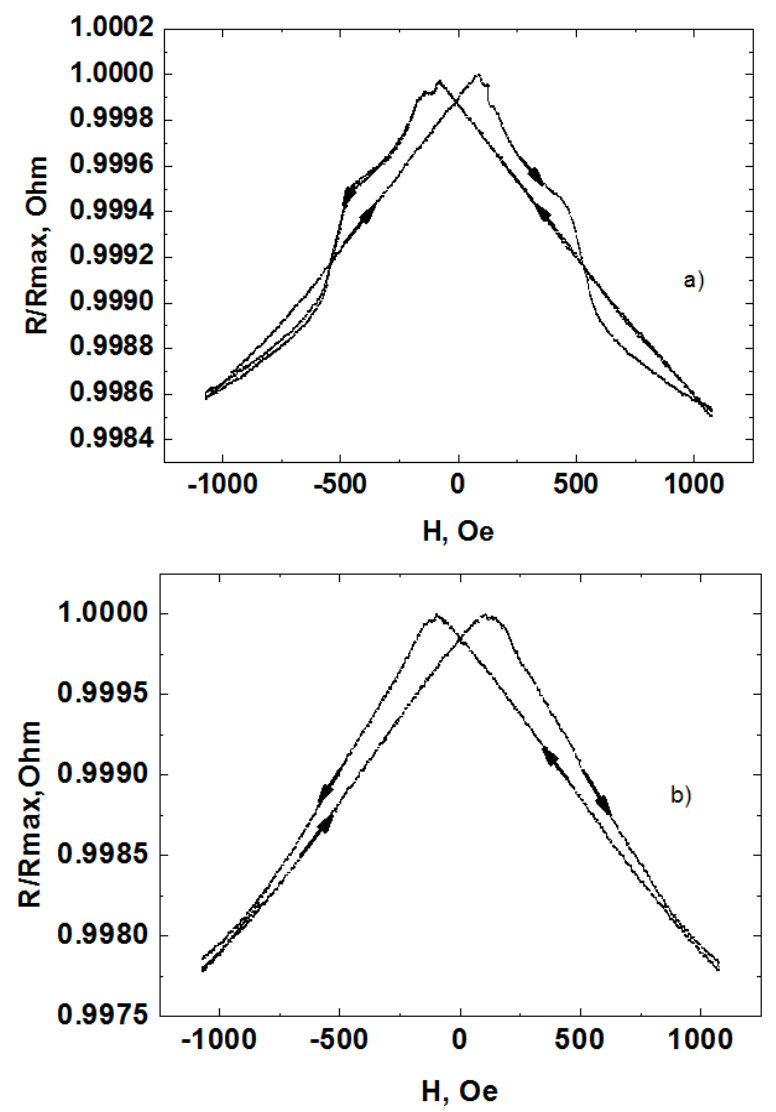

Fig. 5. Dependence of the resistance on magnetic field applied along the hard (a) and easy (b) axes.

Figure 6 shows the difference between magnetoresistances for magnetic field oriented along hard and easy axes. The shape of the functions shown in Fig. 6 reflects the contribution of the interlayer interaction to magnetoresistance. Giant magnetoresistance (GMR) of the heterostructure and anisotropic magnetoresistance (AMR) are proportional to $(\cos \theta)^{2}$ where $\theta$ is the angle between magnetizations of the layers [3]. The features of the magnetoresistance observed for magnetic field applied along the hard axis and caused by switching of the TCFC film magnetization are similar to ones predicted in [4].

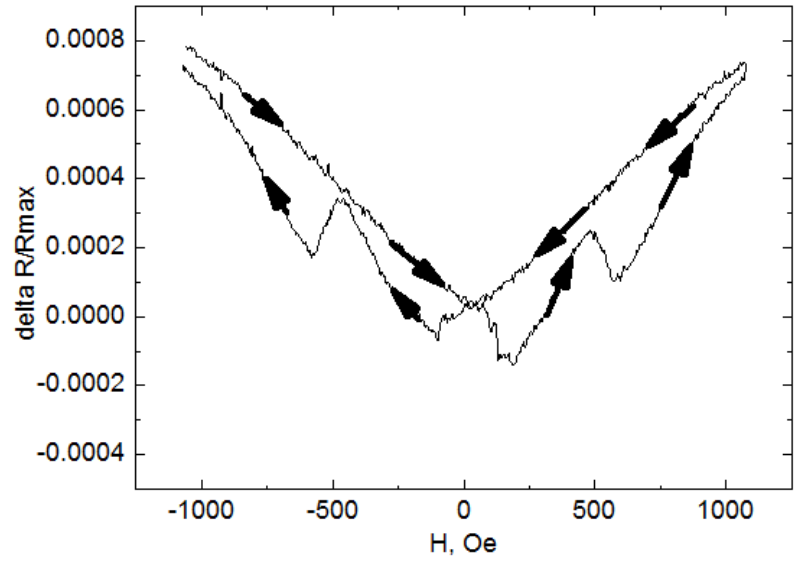

Fig. 6. Difference between magnetoresistance presented on Fig. $5 \mathrm{a}$ and Fig $5 \mathrm{~b}$. The arrows point. The arrows indicate the direction of magnetic field change.

\section{Conclusion}

Experimental data obtained by means of magneto-optical Kerr effect and magnetoresistance and show that the features observed in magnetotransport characteristics are caused by the process of magnetization reversal at the interface between a manganite thin film and an intermetallic superlattice. The magnetic interaction between manganite and intermetallic superlattice is found to be antiferromagnetic.

The authors wish to thank A.M. Petrzhik, A.V. Shadrin, Y.V. Kislinskii and T.A. Shayhulov for help with experiment and useful discussion. Support by International Associated Laboratory LEMAC-LICS, Russian Foundation for Basic Research 16-29-14022, and Scientific School NSh-8168.2016.2 is acknowledged. Samples were partly processed with the support of the RENATECH technological network.

\section{References}

1. D. S. Jeong, R. Thomas, R. S. Katiyar, J. F. Scott, H. Kohlstedt, A. Petraru and Cheol Seong Hwang, Rep. Prog. Phys.75, 076502 (2012).

2. H.J. Mao, P. X. Miao, J. Z. Cong, C. Song, B. Cui, J. J. Peng, F. Li, G. Y. Wang, Y. G. Zhao, Y. Sun, L. R. Xiao, and F. Pan, J. Appl. Phys. 116, 053703 (2014).

3. Y. Dusch, N. Tiercelin, A. Klimov, S. Giordano,V. Preobrazhensky, and Ph. Pernod, J. Appl. Phys. 113, 17C719 (2013).

4. A. Klimov, N. Tiercelin, V. Preobrazhensky, and Ph. Pernod, IEEE Transactions on Magnetics, 42, 3090 (2006).

5. Y. Dusch, V. Rudenko, N. Tiercelin, S. Giordano, V. Preobrazhensky, and P. Pernod, Nanomater. Nanostruct. 2, 44 (2012).

6. K.A. Zvezdin, J. Physics of the Solid State 1, 120125 (2000)

7. A.Aharoni, J. Appl. Phys. 76, 6977 (1994). 